\title{
Factors affecting mortality in patients with COPD exacerbations requiring ICU admission
}

\author{
Chok Limsuwat, Nopakoon Nantsupawat, Elvira Umyarova, Kamonpun Ussavarungsi, \\ and Kenneth Nugent
}

\begin{abstract}
Background: Acute exacerbations of chronic obstructive pulmonary disease (AECOPD) often require hospital admission and have a significant mortality rate. Patients with AECOPD who need intensive care (ICU) have higher mortality rates. Identifying factors associated with increased mortality might change approaches to treatment and improve communication with patients' families about prognosis.
\end{abstract}

Methods: Patients with AECOPD (ICD 9 code 491.21) directly admitted to the ICU between 1/1/2006 and 12/31/2010 were retrospectively reviewed. The inclusion criteria were age 45 years or older, diagnosis of AECOPD, and admission to an ICU. The exclusion criteria included any history of another respiratory disease or decompensated cardiac disease. The primary goal was to determine factors which affect survival.

Result: Two hundred and seventeen patients were included this study. The mean ages were $70.4 \pm 10.4$ years in the in-hospital death group and $66.4 \pm 10.9$ years in the survivors. The overall mortality rate was $12 \%$. Multivariate analysis showed that the mortality rate was significantly associated with a low mean arterial blood pressure (MAP) (odds ratio [OR] 0.91, 95\% confidence interval [Cl] 0.86-0.96), an intubation event (OR 6.12, 95\% Cl 1.24-30.87), and an elevated blood urea nitrogen (BUN) (OR 1.06, 95\% Cl 1.01-1.12) $(P<0.05$ for each factor).

Conclusion: This study identified clinical parameters associated with increased mortality in patients with AECOPD admitted to an ICU. These factors include a low MAP, intubation, and a high BUN and are easily obtained during the initial evaluation of the patient. They reflect the severity of the acute exacerbation and complications in other organ systems.

Key words: COPD, mortality, acute flare, risk factors

\section{INTRODUCTION}

Chronic obstructive pulmonary disease (COPD) is an important disease that causes hospital admissions, disability, high morbidity, and mortality. In the United States, COPD accounts for about 14 million

Corresponding author: Chok Limsuwat, MD Contact Information: chok.limsuwat@ttuhsc.edu DOI : 10.12746/swrccc2013.0102.013 outpatient visits annually; acute exacerbations of COPD (AECOPD) accounted for 725,000 hospitalizations and 1.5 million emergency department visits in 2000. ${ }^{1,2}$ In the United Kingdom it is estimated that $1.5 \%$ of the population has COPD. ${ }^{3}$ Some studies report that three to six percent of AECOPD patients require hospitalization and that mortality ranges from three to $10 \%$ during these admissions. ${ }^{4-8}$ This rate is much higher in the intensive care unit (ICU) setting and approaches $30 \%$ in patients older than $65 .^{9,10}$ 
Comorbidity appears to increase the mortality with AECOPD, and recent studies have focused on clinical parameters which predict inpatient and 30-day mortality following AECOPD. ${ }^{11-14}$ These studies used models with $\mathrm{PaCO} 2$, oxygen saturation ${ }^{15}, \mathrm{BMI}^{16,17}$, age $^{16,18,19}$, and comorbidities to predict in-hospital mortality during AECOPD. ${ }^{19}$ However, these studies did not address mortality in AECOPD patients admitted to ICUs. Identifying factors that strongly affect mortality in ICU patients might help clinicians make better treatment decisions and facilitate discussions with patients and their families about the prognosis.

We hypothesized that underlying medical conditions, such as congestive heart failure (CHF), diabetes mellitus and cancer, nursing home residence, routine laboratory tests on admission, chest X-rays, and medical prediction scores, such as the CURB65 and the APACHE II, might help physicians identify patients at risk for death during admissions to the intensive care unit (ICU). We reviewed the medical records in COPD patients admitted to an ICU to identify factors associated with mortality.

\section{Methods}

We conducted retrospective chart reviews of patients at University Medical Center in Lubbock, TX, between January 1, 2006, and December 31, 2010, diagnosed with a COPD exacerbation who needed ICU admission. We used ICD 9 codes 491.21 (obstructive chronic bronchitis with [acute] exacerbation) to identify patients. The inclusion criteria were ages 45 year or older, a diagnosis of COPD exacerbation defined by at least two criteria (increased dyspnea, cough increased in frequency and severity, and sputum production increased in volume and/or changed character), and patients admitted to the ICU on hospital admission. The exclusion criteria included a history of another respiratory disease, such as asthma, or a cardiac disorder resulting in congestive heart failure. The Institutional Review Board at Texas Tech University Health Sciences Center in Lubbock, TX, approved the study.

From electronic medical records and case management resources, we collected the patients' ages, sex, body mass indices(BMI), baseline pulmonary function tests, comorbidities, CBCs, complete metabolic profiles, initial arterial blood gases, sputum cultures, blood cultures, chest X-rays, APACHE II scores, CURB-65 scores, final diagnoses, length of hospital stays, and in-hospital mortality rates. The primary outcome was the identification factors associated with increased in-hospital mortality. We separated patients into two groups: group one died during hospital admission and group two survived to hospital discharge.

We used descriptive statistics to characterize the study population. We calculated means \pm standard deviations, medians with interquartile ranges, and frequencies with percentages as needed. T-tests and Chi square tests were used to analyze differences between patients who died and patients who survived the acute flare. We then used logistic regression analysis to analyze which factors affected the in-hospital mortality. We selected factors with a P-value less than 0.05 from the univariate analysis to analyze in the multivariate logistic regression models to determine factors that predicted increased mortality. Statistical analysis was performed using SPSS version 16.0; $P$-values $<0.05$ were consider statistically significant.

\section{RESULTS}

We identified 325 patients admitted to intensive care units with the ICD 9 code for AECOPD between January 1, 2005, and January 1, 2011. However, after chart reviews 108 patients were excluded from the study (5 deaths, 103 survivors) because they did not meet inclusion criteria or met exclusion criteria. The majority of excluded patients had congestive heart failure with an exacerbation or acute asthma. Therefore, this study enrolled 217 cases with AECOPD who needed ICU admission; 26 died during hospitalization and 191 survived to hospital discharge (Figure 1). The overall length of stay was $9.01 \pm 6.00$ days; the length of stay was $8.37 \pm 4.99$ days in survivors and $13.69 \pm 9.78$ days in nonsurvivors.

Most patients were elderly with comorbidity; the mean age was $70.4 \pm 10.4$ years and $66.9 \pm 10.9$ years in the mortality and survivor groups, respec- 
Figure 1 Case selection process

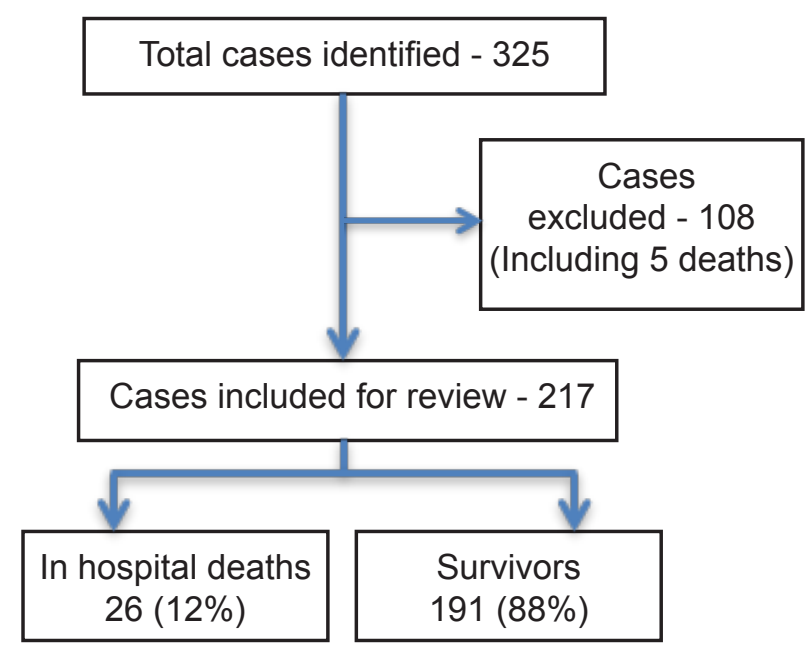

tively (Table1). Forty-seven percent of the patients (102/217) were men. The body mass index was approximately $26 \mathrm{~kg} / \mathrm{m}^{2}$ in both groups. Baseline pulmonary function tests were available in $34.6 \%$ of patients in the mortality group and in $38.7 \%$ of the survivor group; the FEV1s were lower in the mortality group $(P=N S)$. There were no statistically significant differences in baseline characteristics between the mortality and the survivor groups except for nursing home status. Most patients (57.6\%) who died came from nursing homes; $8.3 \%$ of survivors came from nursing homes $(P<0.001)$ (Table 1). We compared nursing home patients with non-nursing home patients and found multiple differences at a P-value of less than 0.1 ; all differences indicated that nursing home patients were older and sicker and had more comorbidity (Table 2).

Univariate logistic regression demonstrated a statistically significant increase in risk of death in patients with low mean arterial pressure, low oxygen saturation, low arterial $\mathrm{pH}$, high blood glucose, low hemoglobin, high BUN, low albumin, pleural effusion, intubation, high APACHE II score, high CURB 65 score, and nursing home residence (Table 3). However, the FEV1, positive blood cultures, positive

Table 1 Demographic data

\begin{tabular}{|l|c|c|c|}
\hline \multicolumn{1}{|c|}{ Variable } & In hospital death & Survivors & P-value \\
\hline Age mean \pm SD, year & $70.4 \pm 10.4$ & $66.9 \pm 10.9$ & 0.71 \\
Men (N=102) & $16(61.5 \%)$ & $86(45 \%)$ & 0.91 \\
BMI \pm SD & $26.1 \pm 7.1$ & $26.7 \pm 8.5$ & 0.55 \\
PFT tests (N=86) & $9(34.6 \%)$ & $74(38.7 \%)$ & \\
FEV1 > 80\% predicted & 0 & 1 & \\
$50 \%<$ FEV1 $<80 \%$ & 1 & 23 & \\
$30 \%<$ FEV1 <50\% & 4 & 18 & \\
FEV1 <30\% & 4 & $55(28.8 \%)$ & 0.82 \\
Underlying diseases & $7(26.9 \%)$ & $111(58.1 \%)$ & 0.61 \\
Diabetes (N=62) & $18(69.2 \%)$ & $5(2.6 \%)$ & 0.44 \\
Hypertension (N=129) & $2(7.6 \%)$ & $16(8.3 \%)$ & 0.77 \\
Lung Cancer (N=7) & $5(19.2 \%)$ & $18(9.4 \%)$ & 0.41 \\
Chronic kidney disease (N=21) & $5(19.2 \%)$ & $44(23 \%)$ & 0.76 \\
Cerebrovascular accident $(\mathrm{N}=23)$ & $9(34.6 \%)$ & $16(8.3 \%)$ & $<0.001$ \\
Congestive heart failure (N=53) & $15(57.6 \%)$ & & \\
Nursing home (N=31) & & & \\
\hline
\end{tabular}

Summary variables are presented as the mean $\pm S D$ for quantitative variable and the count (\%) for discrete variables 
sputum cultures, pulmonary infiltrates on chest x-ray, and alterations in mental status were not significantly associated with outcomes.

Multivariate logistic regression analysis using significant factors identified in the univariate analysis demonstrated that a low mean arterial MAP (OR 0.91, 95\% Cl 0.86-0.96), an intubation event (OR 6.12, 95\%
Cl 1.24-30.87), and an elevated BUN (OR 1.06, 95\% Cl 1.01-1.12) $(P<0.05$ for each factor $)$ significantly increased the odds ratio for mortality (Table 4).We excluded APACHE II scores and CURB-65 scores in this analysis to eliminate the possibility that a composite score would obscure the contribution of individual factors. We also excluded nursing home status for the same reason.

Table 2 Nursing Home Patient Characteristics

\begin{tabular}{|c|c|c|c|}
\hline Factors & $\begin{array}{l}\text { Nursing home } \\
\qquad N=31\end{array}$ & $\begin{array}{l}\text { Non Nursing home } \\
\qquad N=186\end{array}$ & P-value \\
\hline Age mean $\pm S D$, year & $71 \pm 2.4$ & $66.7 \pm 0.8$ & 0.02 \\
\hline Men & 18 of 31 & 82 of 186 & 0.18 \\
\hline BMI \pm SD & $25.5 \pm 1.2$ & $26.9 \pm 0.7$ & 0.42 \\
\hline PFT tests $(\mathrm{N}=86)$ & 7 of 31 & 79 of 186 & \\
\hline FEV1, predicted & $38 \pm 5.1$ & $42.8 \pm 2.0$ & 0.48 \\
\hline \multicolumn{4}{|l|}{ Underlying diseases } \\
\hline Diabetes $(\mathrm{N}=62)$ & 13 of 31 & 49 of 186 & 0.07 \\
\hline Hypertension ( $N=129)$ & 24 of 31 & 105 of 186 & 0.28 \\
\hline Lung Cancer $(\mathrm{N}=7)$ & 1 of 31 & 6 of 186 & $<0.01$ \\
\hline Chronic kidney disease $(\mathrm{N}=21)$ & 4 of 31 & 17 of 186 & 0.04 \\
\hline Cerebrovascular accident $(\mathrm{N}=23)$ & 6 of 31 & 17 of 186 & 0.09 \\
\hline Congestive heart failure $(\mathrm{N}=53)$ & 10 of 31 & 43 of 186 & 0.28 \\
\hline Age $>65(N=131)$ & 24 of 31 & 107 of 186 & 0.04 \\
\hline Mean arterial pressure $(\mathrm{mmHg})$ & $72 \pm 3.8$ & $84.7 \pm 1.3$ & $<0.01$ \\
\hline Initial 02 sat (\%) & $89.3 \pm 1.3$ & $92.3 \pm 0.4$ & $<0.01$ \\
\hline Initial glucose (mg/dl) & $172.9 \pm 16.9$ & $153 \pm 5.4$ & 0.18 \\
\hline Hemoglobin (g/dL) & $11.8 \pm 0.4$ & $13.2 \pm 0.17$ & $<0.01$ \\
\hline 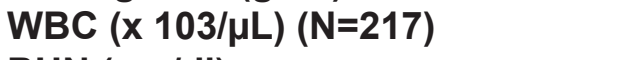 & $14.1 \pm 1.0$ & $12.57 \pm 0.53$ & 0.28 \\
\hline BUN (mg/dl) & $33.9 \pm 4.9$ & $19.3 \pm 0.94$ & $<0.01$ \\
\hline Albumin (g/dL) & $3.1 \pm 0.1$ & $3.7 \pm 0.04$ & $<0.01$ \\
\hline $\mathrm{pH}(\mathrm{ABG})(\mathrm{N}=193)$ & $7.31 \pm 0.02$ & $7.33 \pm 0.01$ & 0.33 \\
\hline \multicolumn{4}{|l|}{ CXR (N=217) } \\
\hline Pleural effusion $(\mathrm{N}=22$ of 217$)$ & 7 of 31 & 15 of 186 & 0.02 \\
\hline Infiltration $(\mathrm{N}=60$ of 217$)$ & 11 of 31 & 49 of 186 & 0.39 \\
\hline Intubation ( $\mathrm{N}=92$ of 217$)$ & 21 of 31 & 71 of 186 & $<0.01$ \\
\hline $\operatorname{BiPAP}(\mathrm{N}=35$ of 217$)$ & 2 of 31 & 33 of 186 & 0.11 \\
\hline APACHE II score $(\mathrm{N}=204)$ & $17.2 \pm 1.4$ & $12.3 \pm 0.4$ & $<0.01$ \\
\hline
\end{tabular}


Table 3 Univariate logistic regression

\begin{tabular}{|c|c|c|c|c|c|}
\hline Factors & $\begin{array}{c}\text { In hospital deaths } \\
(\mathrm{N}=26)\end{array}$ & $\begin{array}{c}\text { Survivors } \\
(\mathrm{N}=191)\end{array}$ & P-value & $\begin{array}{l}\text { Odds } \\
\text { ratio }\end{array}$ & $95 \% \mathrm{Cl}$ \\
\hline \multicolumn{6}{|l|}{ Age } \\
\hline Over all & $70.4 \pm 10.4$ & $66.9 \pm 10.9$ & 0.13 & 1.03 & 0.99-1.07 \\
\hline Age $>65(N=131)$ & 19 of 131 & 112 of 131 & 0.16 & 1.92 & $0.77-4.78$ \\
\hline \multicolumn{6}{|l|}{ Sex } \\
\hline Men $(N=102)$ & 16 of 26 & 86 of 191 & 0.12 & 1.96 & $0.84-4.25$ \\
\hline FEV1, \% predicted ( $\mathrm{N}=86$ of 217 ) & $33.1 \pm 9.5$ & $43.5 \pm 17.8$ & 0.097 & 0.96 & 0.99-1.01 \\
\hline Nursing home $(\mathrm{N}=31$ of 217$)$ & 15 of 26 & 16 of 191 & $<0.001$ & 14.9 & $5.88-38.85$ \\
\hline \multicolumn{6}{|l|}{ Initial clinical and laboratory result } \\
\hline $\mathrm{MAP}^{*}(\mathrm{mmHg})$ & $64.1 \pm 13.4$ & $85 \pm 18.3$ & $<0.001$ & 0.92 & $0.89-0.95$ \\
\hline MAP $<65 \mathrm{mmHg}$ & 15 of $26(58 \%)$ & 27 of $191(14 \%)$ & $<0.001$ & 16.35 & $6.08-43.94$ \\
\hline Initial O2 sat (\%) & $88.1 \pm 8.5$ & $92.3 \pm 4.5$ & $<0.001$ & 0.89 & $0.83-0.95$ \\
\hline Initial BS (mg/dL) & $192 \pm 97$ & $151 \pm 69.6$ & 0.015 & 1.006 & $1.001-1.01$ \\
\hline Hemoglobin (g/dL) & $11.6 \pm 2.6$ & $13.2 \pm 2.2$ & 0.002 & 0.746 & $0.62-0.9$ \\
\hline 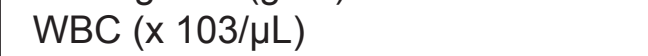 & $13.97 \pm 6.15$ & $12.63 \pm 7.21$ & 0.37 & 1.02 & 0.97-1.07 \\
\hline $\mathrm{BUN}(\mathrm{mg} / \mathrm{dL})$ & $36.5 \pm 28.6$ & $19 \pm 12.7$ & $<0.001$ & 1.05 & $1.03-1.07$ \\
\hline Albumin (g/dL) & $3.1 \pm 0.7$ & $3.7 \pm 0.5$ & $<0.001$ & 0.15 & $0.07-0.34$ \\
\hline $\mathrm{pH}$ form initial $\mathrm{ABG}$ & $7.27 \pm 0.13$ & $7.33 \pm 0.1$ & 0.009 & 0.01 & $0.00-0.28$ \\
\hline \multicolumn{6}{|l|}{ Culture result } \\
\hline Positive blood culture & 4 of $25(16 \%)$ & 10 of $161(6.2 \%)$ & 0.09 & 2.87 & $0.83-10.0$ \\
\hline Positive sputum culture & 6 of $21(28.6 \%)$ & 42 of $151(27.8 \%)$ & 0.94 & 1.04 & $0.38-2.85$ \\
\hline \multicolumn{6}{|l|}{ CXR } \\
\hline Pleural effusion & 7 & 15 & 0.007 & 4.08 & $1.48-11.25$ \\
\hline Infiltration ( $\mathrm{N}=60$ of 217$)$ & 10 & 50 & 0.26 & 1.64 & $0.69-3.85$ \\
\hline Intubation ( $\mathrm{N}=92$ of 217 ) & 21 of $26(80.7 \%)$ & 71 of $191(37.1 \%)$ & $<0.001$ & 7.04 & 2.54-19.49 \\
\hline $\operatorname{BiPAP}(\mathrm{N}=35$ of 217$)$ & $4(15.38 \%)$ & $31(16.23 \%)$ & 0.90 & 0.93 & $0.30-2.89$ \\
\hline \multicolumn{6}{|l|}{ Clinical score } \\
\hline APACHE II score $(\mathrm{N}=204)$ & $21.9 \pm 7$ & $11.7 \pm 4.2$ & $<0.001$ & 1.48 & $1.28-1.7$ \\
\hline CURB-65 score $(\mathrm{N}=215)$ & $3.3 \pm 1$ & $1.6 \pm 1$ & $<0.001$ & 4.04 & 2.47-6.62 \\
\hline
\end{tabular}

Summary variables are presented as the mean $\pm S D$ for quantitative variable and the count (\%) for discrete variables ${ }^{*} M A P=$ Mean arterial pressure

\section{Discussion}

Our study design involved patients with $\mathrm{AE}$ COPD who required ICU admission and used routine admission data (such as age, sex, BMI, underlying medical problems, and nursing home status), diagnostic tests, and laboratory results to identify patients at a higher risk for mortality during the hospital admission. In this study the overall mortality rate for patients with AECOPD requiring ICU admission was $12 \%$ which is slightly lower than the rates reported in other studies which ranged from $15 \%$ to $24 \% .^{9,10}$

Our study demonstrated that patients with AECOPD have lower survival rates if they have low mean 
Table 4 Multivariate logistic regression analysis

\begin{tabular}{|l|c|c|c|c|c|}
\hline \multicolumn{1}{|c|}{ Factors } & $\begin{array}{c}\text { In hospital deaths } \\
(\mathrm{N}=26)\end{array}$ & $\begin{array}{c}\text { Survivors } \\
(\mathrm{N}=191)\end{array}$ & P-value & $\mathrm{B}$ & (OR) $95 \% \mathrm{Cl}$ \\
\hline $\begin{array}{l}\text { Mean arterial pressure } \\
(\mathrm{mmHg})\end{array}$ & $64.1 \pm 13.4$ & $85 \pm 18.3$ & $<0.001$ & -0.09 & $(0.91) 0.86-0.96$ \\
Initial O2 sat (\%) & $88.1 \pm 8.5$ & $92.3 \pm 4.5$ & 0.16 & -0.66 & $(0.94) 0.85-1.03$ \\
Initial glucose (mg/dL) & $192 \pm 97$ & $151 \pm 69.6$ & 0.60 & -0.02 & $(0.99) 0.99-1.01$ \\
Hemoglobin (g/dL) & $11.6 \pm 2.6$ & $13.2 \pm 2.2$ & 0.14 & -0.22 & $(0.81) 0.60-1.07$ \\
BUN (mg/dL) & $36.5 \pm 28.6$ & $19 \pm 12.7$ & 0.04 & 0.06 & $(1.06) 1.01-1.12$ \\
Albumin (g/dL) & $3.1 \pm 0.7$ & $3.7 \pm 0.5$ & 0.35 & -0.55 & $(0.58) 0.18-1.83$ \\
pH & $7.27 \pm 0.13$ & $7.33 \pm 0.1$ & 0.819 & -0.71 & $(0.49) 0.01-221$ \\
Pleural effusion & 7 of 26 & 15 of 181 & 0.09 & 1.65 & $(5.2) 0.76-35.86$ \\
Intubation & 21 of 26 & 76 of 191 & 0.03 & 1.82 & $(6.12) 1.24-30.87$ \\
& $(80.7 \%)$ & $(39.7 \%)$ & & & \\
\end{tabular}

arterial pressures, high BUNs, or require intubation. Survival was significantly increased with an increase in mean arterial pressure, and our multivariate logistic regression indicated that a one $\mathrm{mmHg}$ increase in blood pressure decreased mortality by $9 \%$. MAP is a clinical parameter that reflects hemodynamic status, and a lower MAP should imply more disease severity. A high blood urea nitrogen was also associated with higher mortality in our study. BUN is a component of the CURB-65 which predicts the severity of pneumonia and the BAP-65 which predicts poor outcomes in AECOPD. 20,21,22 High BUNs may reflect impaired circulation and/or acute kidney injury which could lead to poor outcomes. We also tested the utility of the CURB-65 score to predict mortality since a previous study showed that this score could predict early mortality in AECOPD. ${ }^{21,22}$ Both the APACHE II score and the CURB-65 score predicted mortality in our study in the univariate analysis, but we did not include these factors in the multivariate logistic regression since these composite scores could potentially obscure the contribution of single factors. Patients in our study who needed intubation had a mortality rate of $21.6 \%$ (21 of 97), which was significantly higher than in patients who did not require intubation on admission and had a mortality rate of $4.2 \%$ (5 of 120 ). This reflects the severity of the underlying COPD, and/or the severity of the acute flare in disease, and/or the potential for ventilator associated adverse events.

Low albumin levels were also associated with increased mortality in the univariate analysis, and this is consistent with an earlier study which used routine laboratory tests to help predict in-hospital mortality in AECOPD. ${ }^{23}$ Both low hemoglobins and low albumin levels may represent underlying malnutrition prior to admission and/or systemic inflammation, and these processes could contribute to poor hospital outcomes. ${ }^{24,25}$ Patients in nursing homes usually have either an acute illness that requires convalescence, or a chronic medical illness with significant impairment, or both. Nursing home status had the highest odds ratio in the univariate analysis and is a simple predictor for poor outcomes. ${ }^{26}$

Our study is limited by the fact that it was a retrospective study and used the medical records for all clinical information. In addition, our ICU has an open admission policy, and physicians may not use uniform admitting criteria or approaches to treatment. Therefore, the patients in this ICU may differ from other ICUs. The strength of this study is that we used only routine admission data to predict mortality risk factors in AECOPD. Therefore, these results are simple and easy to use at the time of admission. Moreover, the important factors identified in this study represent 
either quantitative information that has reasonable measurement accuracy or categorical information with a clear yes/no answer.

\section{Conclusions}

We identified several clinically important parameters which are easily available in ICU patients and suggest that these simple parameters (low MAP, intubation, and high BUN) can help predict in-hospital mortality in patients with AECOPD requiring ICU admission. Identifying high risk patients may allow interventions to reduce the mortality rate in this patient group. For example, a more consistent effort to use BiPAP might avoid intubation and reduce mortality. In addition, this risk assessment should help clinicians inform patients and their families about prognosis and care expectations while the patient is in the ICU.

\section{KEY POINTS}

1. COPD patients with acute exacerbations have a $12 \%$ mortality rate if admitted to an ICU.

2. Nursing home patients with COPD often have multiple comorbidities.

3. Patients with low mean blood pressures, high BUNs, and severe respiratory failure requiring intubation have higher mortality rates.

\begin{abstract}
Author Affiliation: Chok Limsuwat, MD, and Elvira Umyarova, MD, are residents in the Department of Internal Medicine at TTUHSC, Nopakoon Nantsupawat, MD, is a research assistant in the Department of Internal Medicine at TTUHSC, Kamonpun Ussavarungsi, MD, is a fellow in pulmonary and critical care medicine at the Mayo Clinic Florida, 4500 San Pablo Road, Jacksonville, FL 32224, Kenneth Nugent, MD, is a pulmonary physician in the Department of Internal Medicine at TTUHSC.
\end{abstract}

Received: $1 / 10 / 2013$

Accepted: 3/26/2013

Reviewers: Sandra G Adams, MD

Published electronically: 4/16/2013

Conflict of Interest Disclosures: None

\section{REFERENCES}

1. Murphy SL. Deaths: final data for 1998. Natl Vital Stat Rep 2000; 48:1-105.

2. Mannino DM, Homa DM, Akinbami LJ, Ford ES, Redd SC. Chronic obstructive pulmonary disease surveillance--United States, 1971-2000. MMWR Surveill Summ. 2002 Aug 2; 51(6):1-16.

3. Chronic obstructive pulmonary disease. National clinical guideline on management of chronic obstructive pulmonary disease in adults in primary and secondary care. Thorax 2004; 59 Suppl 1:1-232.

4. Seemungal TA, Donaldson GC, Paul EA, Bestall JC, Jeffries DJ, Wedzicha JA. Effect of exacerbation on quality of life in patients with chronic obstructive pulmonary disease. Am J Respir Crit Care Med 1998; 157:1418-22.

5. Miravitlles M, Murio C, Guerrero T, Gisbert R. Pharmacoeconomic evaluation of acute exacerbations of chronic bronchitis and COPD. Chest 2002; 121:1449-55.

6. Groenewegen KH, Schols AM, Wouters EF. Mortality and mortality-related factors after hospitalization for acute exacerbation of COPD. Chest 2003; 124:459-67

7. Mushlin AI, Black ER, Connolly CA, et al: The necessary length of hospital stay for chronic pulmonary disease. JAMA 1991, 266:80-83.

8. Connors AF, Dawson NV, Thomas C, Harrell FE, Desbiens N, Fulkerson WJ, Kussin P, Bellamy P, Goldman L, and Knaus WA. Outcomes of acute exacerbations of severe chronic obstructive lung disease. Am J Resp Crit Care Med 1996; 154:959-967.

9. Afessa B, Morales IJ, Scanlon PD, Peters SG. Prognostic factors, clinical course, and hospital outcome of patients with chronic obstructive pulmonary disease admitted to an intensive care unit for acute respiratory failure. Crit Care Med 2002; 30:1610-5.

10. Seneff MG, Wagner DP, Wagner RP, Zimmerman JE, Knaus WA. Hospital and 1-year survival of patients admitted to intensive care units with acute exacerbation of chronic obstructive pulmonary disease. JAMA 1995; 274:1852-7.

11. Parappil A, Depczynski B, Collett P, Marks GB. Effect of comorbid diabetes on length of stay and risk of death in patients admitted with acute exacerbations of COPD. Respirology 2010; 15:918-22.

12. Roche N, Zureik M, Soussan D, Neukirch F, Perrotin D. Predictors of outcomes in COPD exacerbation cases presenting to the emergency department. Eur Respir J 2008; 32:95361 .

13. Faustini A, Marino C, D'Ippoliti D, Forastiere F, Belleudi V, Perucci CA. The impact on risk-factor analysis of different mortality outcomes in COPD patients. Eur Respir J 2008; 32:629-36. 
14. Dransfield MT, Rowe SM, Johnson JE, Bailey WC, Gerald LB. Use of beta blockers and the risk of death in hospitalized patients with acute exacerbations of COPD. Thorax 2008; 63:301-5.

15. Sukumalchantra Y, Dinakara P, Williams MH, Jr. Prognosis of patients with chronic obstructive pulmonary disease after hospitalization for acute ventilatory failure: a threeyear follow-up study. Am Rev Respir Dis 1966; 93:215-22. 16. Connors AF, Jr., Dawson NV, Thomas C, Harrell FE, Jr., Desbiens N, Fulkerson WJ, et al. Outcomes following acute exacerbation of severe chronic obstructive lung disease. The SUPPORT investigators (Study to Understand Prognoses and Preferences for Outcomes and Risks of Treatments). Am J Respir Crit Care Med 1996; 154:959-67.

17. Gray-Donald K, Gibbons L, Shapiro SH, Macklem PT, Martin JG. Nutritional status and mortality in chronic obstructive pulmonary disease. Am J Respir Crit Care Med 1996; 153:961-6.

18. Fuso L, Incalzi RA, Pistelli R, Muzzolon R, Valente $S$, Pagliari G, et al. Predicting mortality of patients hospitalized for acutely exacerbated chronic obstructive pulmonary disease. Am J Med 1995; 98:272-7.

19. Antonelli Incalzi R, Fuso L, De Rosa M, Forastiere F, Rapiti E, Nardecchia B, et al. Co-morbidity contributes to predict mortality of patients with chronic obstructive pulmonary disease. Eur Respir J 1997; 10:2794-800.

20. Lim WS, van der Eerden MM, Laing R, Boersma WG, Karalus N, Town GI, et al. Defining community acquired pneumonia severity on presentation to hospital: an international derivation and validation study. Thorax 2003; 58:37782.

21. Chang CL, Sullivan GD, Karalus NC, Mills GD, McLachlan JD, Hancox RJ. Predicting early mortality in acute exacerbation of chronic obstructive pulmonary disease using the CURB65 score. Respirology 2011; 16:146-51.

22. Shorr AF, Sun X, Johannes RS, Yaitanes A, Tabak YP. Validation of a novel risk score for severity of illness in acute exacerbations of COPD. Chest 2011; 140:1177-83.

23. Asiimwe AC, Brims FJ, Andrews NP, Prytherch DR, Higgins BR, Kilburn SA, et al. Routine laboratory tests can predict in-hospital mortality in acute exacerbations of COPD. Lung 2011; 189:225-32.

24. Aziz EF, Javed F, Pratap B, Musat D, Nader A, Pulimi $\mathrm{S}$, et al. Malnutrition as assessed by nutritional risk index is associated with worse outcome in patients admitted with acute decompensated heart failure: an ACAP-HF data analysis. Heart Int 2011; 6(1):e2.

25. Pardo Cabello AJ, Bermudo Conde S, Manzano Gamero MV. [Prevalence and factors associated to malnutrition in patients admitted to a medium-long stay hospital]. Nutr Hosp 2011; 26:369-75.

26. Landi F, Liperoti R, Fusco D, Mastropaolo S, Quattrociocchi D, Proia A, et al. Sarcopenia and Mortality among Older Nursing Home Residents. J Am Med Dir Assoc 2012; 13: 121-26. 